\title{
Stage-specific protein-domain mutational profile of invasive ductal breast cancer
}

\author{
Ting Yu ${ }^{1,4}$, Kwok Pui Choi $^{1,2}$, Ee Sin Chen ${ }^{3}$ and Louxin Zhang ${ }^{1,4^{*}}$ \\ From The 18th Asia Pacific Bioinformatics Conference \\ Seoul, Korea. 18-20 August 2020
}

\begin{abstract}
Background: Understanding the mechanisms underlying the malignant progression of cancer cells is crucial for early diagnosis and therapeutic treatment for cancer. Mutational heterogeneity of breast cancer suggests that about a dozen of cancer genes consistently mutate, together with many other genes mutating occasionally, in patients.

Methods: Using the whole-exome sequences and clinical information of 468 patients in the TCGA project data portal, we analyzed mutated protein domains and signaling pathway alterations in order to understand how infrequent mutations contribute aggregately to tumor progression in different stages.

Results: Our findings suggest that while the spectrum of mutated domains was diverse, mutations were aggregated in Pkinase, Pkinase Tyr, Y-Phosphatase and Src-homology 2 domains, highlighting the genetic heterogeneity in activating the protein tyrosine kinase signaling pathways in invasive ductal breast cancer.

Conclusions: The study provides new clues to the functional role of infrequent mutations in protein domain regions in different stages for invasive ductal breast cancer, yielding biological insights into metastasis for invasive ductal breast cancer.
\end{abstract}

Keywords: Cancer genes, Integrative analysis, Invasive ductal breast cancer, Stage specific genetic alteration

\section{Background}

Breast cancer is one of over 200 forms of cancer catalogued so far. Only lung cancer accounts for more deaths than breast cancer in women [1]. The majority of in situ breast cancers begin in ducts that connect the lobules to the nipple, twenty to fifty percent of which progress into invasive cancer if they are left untreated in 2016 [2]. Molecular characterizations of the malignant progression

\footnotetext{
*Correspondence: matzlx@nus.edu.sg

'Department of Mathematics, National University of Singapore, 10 Lower Kent Ridge Road, 119076 Singapore, Singapore

${ }^{4}$ Computational Biology Programme, National University of Singapore, 8

Medical Drive, 117596 Singapore, Singapore

Full list of author information is available at the end of the article
}

of ductal carcinoma in situ have been under investigation in the past two decades [3-8]. However, the mechanisms that drive progression are still unclear for ductal carcinoma in situ $[4,9]$.

Treatment options for breast cancer and prognosis depend mainly on tumor subtype and staging. The most widely used staging system classifies breast cancer into five (0 to IV) stages in terms of tumor size, whether or not the tumor has spread to the lymph nodes and the extent of metastasis [10]. Normal breast cells contain receptors that bind to the reproductive hormones estrogen and progesterone, whereas breast tumor cells may contain both, one, or neither of these receptors. Considering the presence or absence of receptors results in classification of 
breast cancers into four therapeutic groups in clinical settings [11]: estrogen receptor-positive $(E R+)$, progesterone receptor-positive $(\mathrm{PR}+)$, HER2 amplified (HER2+) and triple negative. Perou et al. further refined this classification scheme by grouping breast cancers into four PAM50 subtypes based on the microarray expression profile of their DNA [12]: Luminal A, Luminal B, HER2-enriched and basal-like.

Individual breast cancer tumors most often carry consistent alterations of several classic oncogenes (e.g. AKT1, GATA3, PIK3CA) and tumor-suppressive genes (e.g. MAP3K1, PTEN, TP53), along with many other infrequent changes about which little is known [13-15]. Therefore, discerning which and how infrequent mutations contribute through collaboration to tumor progression is crucial $[16,17]$. To meet this challenge, researchers have developed a plethora of methods for detecting cancerassociated infrequent mutations and their effects on cell signaling [18-20] (see also [21] for a review). A study along this line is expected to facilitate identification of biomarkers for early diagnosis of invasive cancers and development of combination therapies targeting specific biological pathways.

Despite the potential clinical significance, stage-specific molecular features of invasive ductal breast cancer (IDBC) remain elusive $[9,22]$. Leveraging the large Phase II dataset for IDBC in The Cancer Genome Atlas (TCGA), we investigated the stage specific molecular features of this cancer. By analyzing the multi-dimensional genomic profiles, along with clinical information, from a cohort of 468 patients, we identified: (1) six novel candidate genes for IDBC (MAP2K4, ZNF384, CFBF, NOCA3, MAP3K4 and RB1), for which evidence has been absent or equivocal; (2) Src-homology 2 (SH2) domain and 21 other protein domains which carry putative driver mutations of low frequency. This study yields biological insights into malignant progression for IDBC and may assist future precision medicine efforts.

\section{Results}

We investigated the whole-exome sequences of 468 patients with IDBC (Stage I: 87, Stage II: 297 and Stage III-IV: 92) and 112 patients with invasive lobular breast cancer (ILBC). In total, 31,242 mutations of different types were detected in 12,358 human genes (Table S1). The inter-individual mutational heterogeneity of IDBC is well indicated by the distribution of the number of genes that mutated in exactly $k$ patients, which has a long low-frequency tail (Fig. S1a). More precisely, only 10 genes (TP53, PIK3CA, GATA3, MAP3K1, MUC16, KMT2C, MUC12, SPEN, MUC4) were each found to be mutated in more than 30 patients and another 20 genes in 20 to 30 patients, whereas over 10,000 genes carried mutations (including missense/nonsense base substitution, frame shift indels and four other types of mutation) in three patients or fewer.

This inter-individual mutational heterogeneity clearly suggests that there are driver mutations of low mutational

Table 1 Functions and mutational specificities of novel cancer-associated genes in IDBC

\begin{tabular}{|c|c|c|c|c|}
\hline \multirow[t]{2}{*}{ Genes } & \multirow[t]{2}{*}{ Canonical functions } & \multirow[t]{2}{*}{ Cancer relevance } & \multicolumn{2}{|c|}{ Specificity } \\
\hline & & & Stage & In situ \\
\hline \multirow[t]{3}{*}{ CFBF } & The encoded transcription factor & Function in & Stage I & No \\
\hline & regulates RUNX and other genes & aberrant estrogen & & \\
\hline & specific to hematopoiesis & receptor signaling & & \\
\hline MAP2K4, & The encoded kinases belong to a & Evading apoptosis, & No & Duct \\
\hline \multirow[t]{4}{*}{ MAP3K4 } & protein kinase signal transduction & Dysfunction of DNA & Stage & Duct \\
\hline & cascade, which can activate the & repair mechanism & & \\
\hline & stress-induced P38 and JNK & & & \\
\hline & MAPK pathways & & & \\
\hline \multirow[t]{3}{*}{ NCOA3 } & The encoded protein (AIB1) & Biomarker for & No & No \\
\hline & enhances estrogen-dependent & evaluating & & \\
\hline & transcription & tamoxifen [24] & & \\
\hline \multirow[t]{3}{*}{ RB1 } & RB1 prevents excessive cell growth & A tumor suppressor & No & Duct \\
\hline & by inhibiting cell cycle progression & that controls cell & & \\
\hline & until a cell is ready to divide & growth [25] & & \\
\hline \multirow[t]{3}{*}{ ZNF384 } & The encoded transcription factor & Involved in cell & Stage I & Duct \\
\hline & involved in extracellular matrix & proliferation in & & \\
\hline & remodeling & other cancers & & \\
\hline
\end{tabular}


frequency and these infrequent driver mutations may likely contribute through collaboration to tumor growth and progression. As such, we investigated not only novel cancer genes of low mutation frequency (Table 1), but also protein domains that were significantly altered by aggregation of infrequent mutations.

\section{Cancer genes carrying stage-specific mutations in IDBC} MutSigCV2 reported 12 out of 12,358 mutated genes at a false discovery rate (FDR) with $q$-value $\leq 0.1$ (Table S2), which examines the gene-specific abundance of mutations relative to the background mutation rate, mutation conservation and clustering [18]. Among these were six functionally established cancer genes AKT1, GATA3, MAP3K1, PIK3CA, PTEN and TP53 (for each $q$-value $\leq 10^{-13}$ ). The other six reported genes that have less clear functions in breast cancer are CBFB, MAP2K4, MAP3K4, NCOA3, RB1 and ZNF384, whose biological functions and cancer relevance are summarized in Table 1. CBFB and ZNF384 are cancer genes for leukemia; RB1 is a gene for retinoblastoma, sarcoma and small-cell lung cancers and MAP3K4 is a gene for pancreatic, breast and colorectal cancers, as listed on the Cancer Gene Census [23]. Mutations that occurred in these genes are illustrated in Fig. S2 and Fig. S3.

All except for NCOA3 (4.9\% versus $4.9 \%$ ) and CBFB (2.56\% versus $2.68 \%)$ mutated significantly more frequently in IDBCs than in 112 patients with ILBC: MAP2K4 (4.7\% versus $1.6 \%, P$-value $\left.<10^{-5}\right)$, RB1 $(4.1 \%$ versus $0.8 \%, P$-value $\left.=1.5 \times 10^{-8}\right)$, ZNF384 $(4.3 \%$ versus $1.6 \%, P$-value $\left.=9.2 \times 10^{-5}\right)$ and MAP3K4 $(2.3 \%$ versus $1.2 \%, P$-value $=0.028)$, neither of which were reported by MutSigCV2 when only 112 ILBC genomes were used.

The mutation coverage (MC) of a gene is the percentage of patients carrying mutations in the gene, also called mutation prevalence [26]. The MCs did not vary much across the stages for TP53, PIK3CA, GATA3, and MAP3K1 (Fig. 1a). However, the MCs of AKT1, CBFB, MAP3K4 and ZNF384 in Stage I were at least 1.5 times as high as in other stages, having $P$-values of $5.27 \times 10^{-3}$, $2.7 \times 10^{-2}, 5.09 \times 10^{-2}, 4.02 \times 10^{-2}$ for enrichment in Stage I (Fig. 1b).

\section{2 protein domains were recurrently hit by infrequent mutations}

Protein domains are evolutionarily and structurally conserved units with specific functions in protein sequences. Tallying mutations in the domain-coding regions in all genes containing a specific protein domain allow us to identify mutations that are rare at the gene level, but occur recurrently within the protein domain $[27,28]$. Therefore, a domain-based approach enables the discovery of new alterations that confer functional advantage to cancer cell development but are not detected through gene-by-gene analysis.

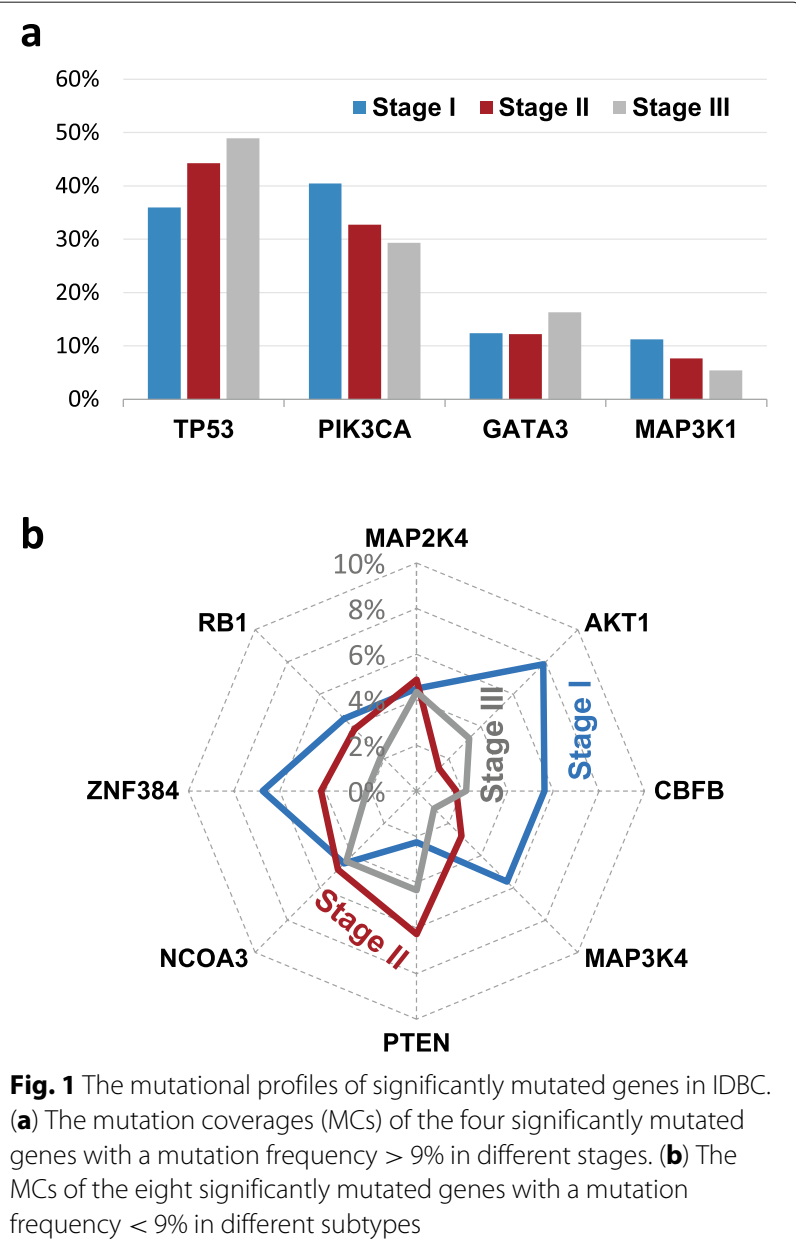

In the 468 patients with IDBC, 4,793 mutations occurred in genomic sequences that encompass the encoding regions of 1,217 (PfamA) protein domains in 3,625 genes (Table S3). 22 protein domains were found to have mutations being distributed in at least 30 genomes (6.4\%), resulting in a $P$-value of less than 0.05 and a normalized Shannon entropy of at least 0.71 (Fig. S4). 18 of these protein domains are found in cancer genes (based on the Cancer Gene Census [23]), whereas supporting evidences for involvement in breast cancer have been reported for the other four in the literature [29, 30]. In addition, 11 of these protein domains were also reported by Yang et al. in their integrative analysis of pan-cancer genomes [28] (Fig. 2a). (These 22 protein domains are functionally annotated in Table S4.)

The mutational spectra of the 22 protein domains were examined in terms of survival status (Fig. 2b) and stages (Fig. 2c). Mutations in both the I-set and fn3 domains are enriched in the group of patients who died with $P$ values of 0.056 and 0.047 , respectively. Additionally, they were depleted in Stage II $\left(P\right.$-values $\left.<2.2 \times 10^{-16}\right)$. The Kaplan-Meier survival analysis further showed that 
a

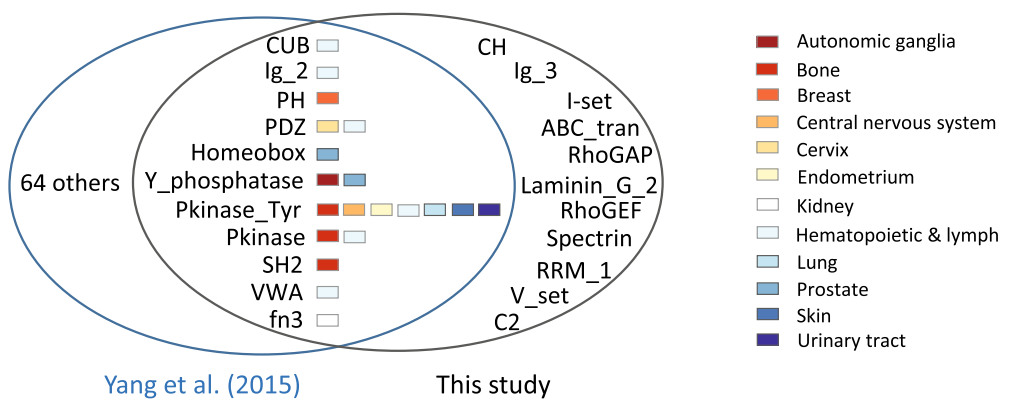

b

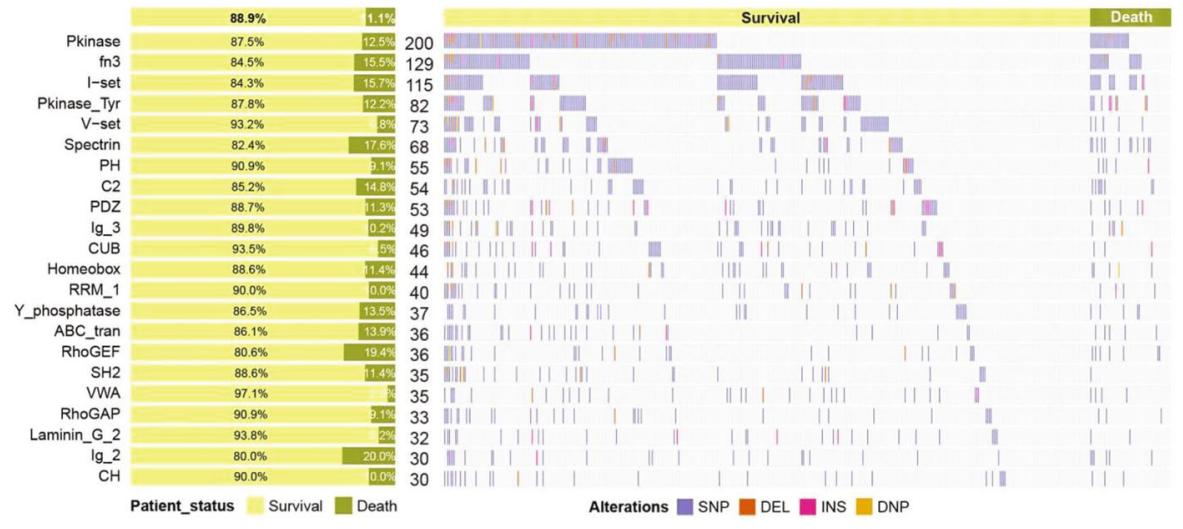

C

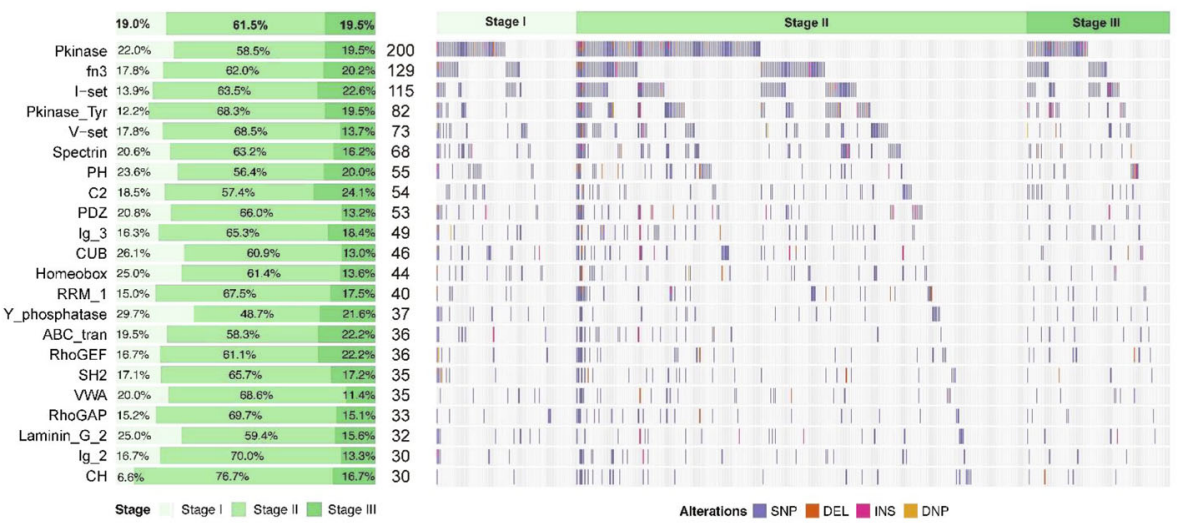

d
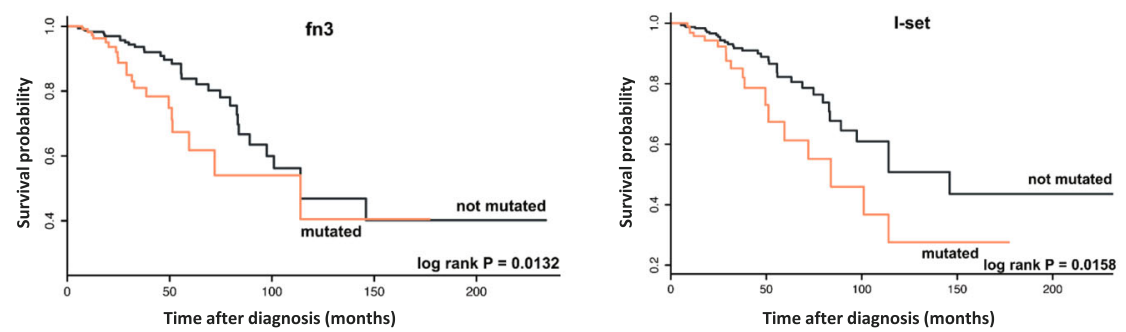

Fig. 222 significantly mutated protein domains and their mutational spectra. (a) 11 reported protein domains were also identified by Yang et al. [28] for other cancers. Here, "others" represent those protein domains that were only reported in [28]. (b) and (c) The distributions of mutations in the 22 protein domains in terms of survival status and staging. Left panel: The percentage of surviving (and deceased) patients (resp. patients in a stage) in which each protein domain is mutated. Right panel: The distribution of the four types of mutations in the groups of patients for the 22 protein domains. Middle column: The number of patients in which a protein domain is mutated. Of note, none of these protein domains was mutated in the patients listed at the end of each group. SNP, single nucleotide polymorphism; DEL, deletion; INS, insertions; DNP, double nucleotide polymorphism. (d) The Kaplan-Meier survival analysis for protein domains fn3 and I-set 
mutation in these two protein domains agree with the poor survival outcomes (cutoff $P$-value $=0.05$, log rank test, Fig. 2d), as well as Spectrin, Ig_2, and RhoGEF domains (Fig. S5). The hazard ratio (HR) analysis also suggests this fact. HR of I-set and fn3 are 2.0473 and 2.0711, respectively, corresponding to $P$-values 0.0144 and 0.0112 .

\section{Critical mutations in $\mathrm{SH} 2$ domains}

Of the 22 protein domains, Pkinase, Pkinase_Tyr, YPhosphatase and Src-homology 2 (SH2) play important roles in protein tyrosine kinase (PTK) signaling pathways. The Pkinase domains were found to be mutated 374 times in 186 kinase genes in 200 patients. The Pkinase_Tyr domain mutated 115 times in 67 PTK genes, including 10 cancer genes [23]. Mutations within the Pkinase_Tyr domain were also found recurrently in breast and seven other cancers. Together with tyrosine kinases, 107 protein tyrosine phosphatases (also called Y-phosphatases) regulate a plethora of cellular processes, including cell growth and oncogenic transformation. Therefore, it is not surprising that the Y-phosphatase domain were also found to highly mutate in the 468 patients.

SH2 domains are critical mediators found in 96 functionally different human proteins [31]. SH2 mutations could lead to constitutive dysfunction of kinase/SH2 domains or the upstream and downstream rewiring of the PTK signaling pathways, as outlined by Creixell et al. recently [16]. The folded structure of SH2 consists of a central anti-parallel three-stranded $\beta$-sheet flanked by two $\alpha$-helices (Fig. 3a); each instance of $\mathrm{SH} 2$ binds phosphotyrosine-containing sequences with high affinity and specificity with two binding pockets [32]. Two R residues, $\mathrm{R} \alpha \mathrm{I} 2$ and $\mathrm{R} \beta \mathrm{B} 5$, play particularly crucial roles in phosphotyrosine binding with further interactions with three residues at Positions $\beta \mathrm{D} 2$ to $\beta \mathrm{D} 4$ (Fig. 3b). Here, we examined $\mathrm{SH} 2$ mutations in pan cancers available in the COSMIC database [33]. Our pan-cancer analysis showed that mutation rarely occurred at $\alpha \mathrm{I} 2$ and $\beta \mathrm{B} 5$ but recurrently occurred at one position $\mathrm{C}$-terminal to them, as well as at the first position in the BC loop. For IDBC, a PTPN11 mutation occurred at $\alpha \mathrm{I} 2$; two mutations in STAT4 and SRMS occurred at $\beta \mathrm{B} 4$ and $\beta \mathrm{B} 5$, respectively; and three mutations in ABL2, P85 $\alpha$ and TEC occurred at $\beta \mathrm{D} 4$.

The binding pocket for residues that lie three to five amino acids $C$-terminal to the $\mathrm{pY}$ involves residues on the opposite face of the central sheet, the EF turn and $\alpha \mathrm{II}$ (Fig. 3a). The PTPN11 and STAT3 mutations recurrently occur near this pocket. For IDBC, five mutations in ABL2, P85 $\beta$, PLC $\gamma 2$, SHD and SRMS were found in $\alpha$ II (Fig. 3c). Since SH2 must obtain sufficient binding energy from the recognition of nearby residues to achieve a high degree of binding specificity, these identified mutations are likely to affect the binding affinity of $\mathrm{SH} 2$ and hence to have critical roles in IDBC, as exemplified by BTK [34] and PTPN11 [35].

\section{Alteration of ERBB2 signaling pathway is beyond HER2}

$\mathrm{SH} 2$ mutations could lead to constitutive dysfunction of kinase/SH2 domains or the upstream and downstream rewiring of the PTK signaling pathways. To validate whether PTK signaling pathways were likely interrupted or not, we conducted a network analysis of the mutational and amplification profiles of IDBC using the HotNet2 program [19]. Our network analysis identified 11 protein complexes and signaling pathways that were significantly mutated (Table S6), including the ERBB2 signaling pathway, the roles of which in breast cancer have been studied extensively. The ERBB2 signaling pathway were altered by infrequent mutations aggregately (Fig. S6).

\section{Discussion}

Cancer is intimately associated with mutations in cancer genes. Because of the vast heterogeneity of breast tumors [13], characterizing genetic alterations associated with the malignant progression of cancer cells remains a great challenge. We set out to resolve this issue for IDBC by investigating stage-specific genetic alterations caused by infrequent mutations using 468 whole-exome sequences and related clinical information. Our study of genetic alterations complements well recent studies of the gene expression signatures of IDBC $[3-5,22]$ and the molecular portraits of invasive lobular breast cancer [36]. It yields biological insights into the malignant progression of IDBC and reveals potential new avenues for seeking targeted agents in breast cancer.

We found six novel candidate genes of low mutation rate for IDBC: CBFB, MAP2K4, MAP3K4, NCOA3, RB1 and ZNF384, for which evidence has been absent or equivocal. Infrequent mutations in CBFB, MAP3K4 and ZNF384 are enriched in Stage I, together with classic breast cancer gene AKT1 (Fig. 1b). This suggests the hypothesis that these four genes of low mutation rate play Stage I-specific roles, whereas classic cancer genes MAP3K1, PTEN, TP53, GATA3 and PIK3CA are important in the entire malignant progression of IDBC.

We further identified 22 recurrently mutated protein domains by tallying infrequent mutations across the patients, most of which are contained in the genes in the Cancer Gene Census. These protein domains include the Pkinase, Pkinase_Tyr, Y-Phosphatase and SH2 domains that play important roles in the PTK signaling pathways. In particular, $\mathrm{SH} 2$ mutations could lead to constitutive dysfunction of kinase/SH2 domains or the upstream and downstream rewiring of the PTK signaling pathways, as outlined by Creixell et al. recently [16]. Therefore, our investigation of $\mathrm{SH} 2$ mutations facilitates identification of 
a

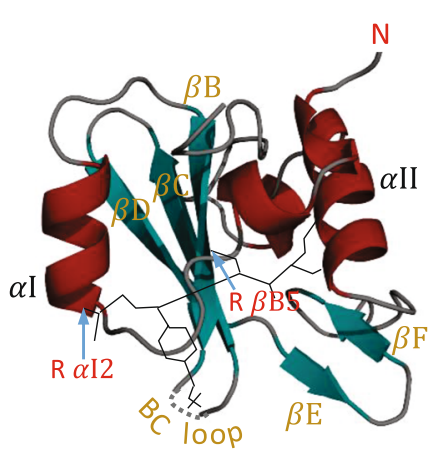

d

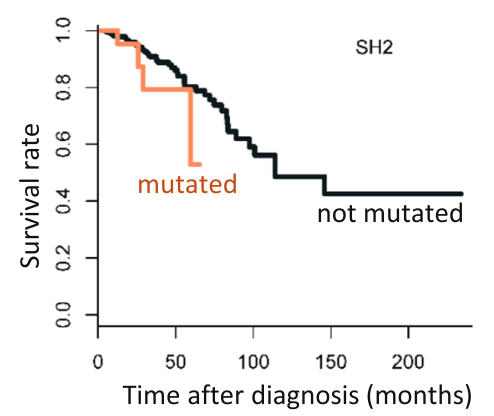

b

SH2 Mutations in Pan-Cancers

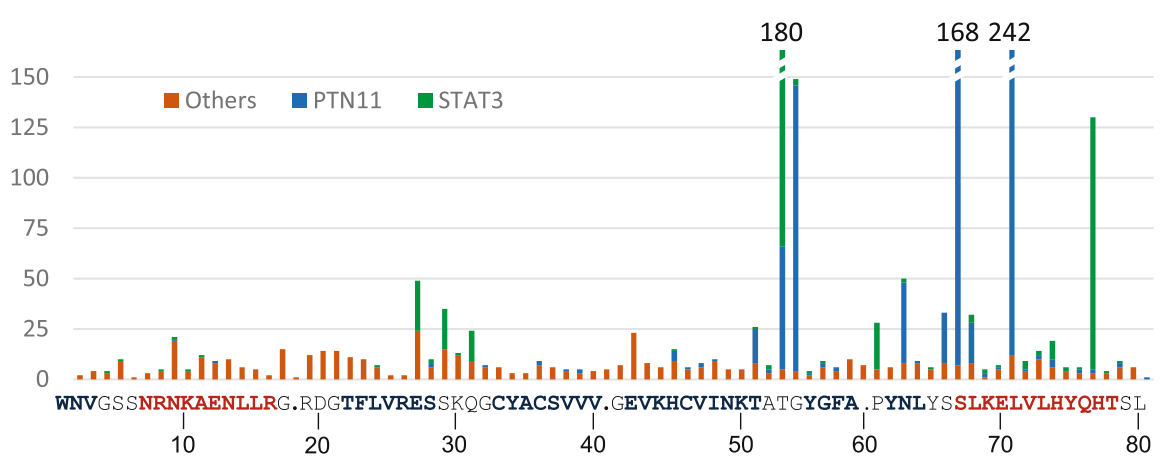

C

SH2 Mutations in IDBC

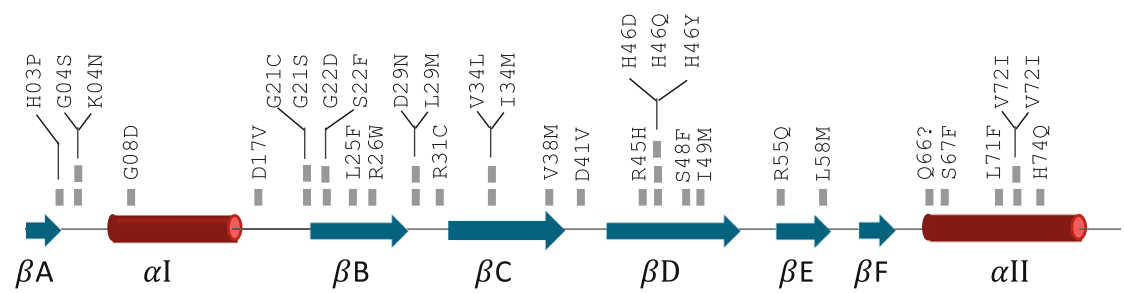

Fig. $3 \mathrm{SH} 2$ mutations in IDBC and pan-cancers. (a) The folded SH2 comprises a central anti-parallel sheet, consisting of the three $\beta$-strands ( $\beta \mathrm{B}, \beta \mathrm{C}$, and $\beta \mathrm{D}$, cyan), sandwiched between two $\alpha$-helices ( $\alpha$ l and $\alpha \|$, dark red). The bound phosphopeptide (thin dark lines) straddles the sheet in such a manner that the phosphotyrosine ( $p Y$ ) binding pocket lies on one side, whereas the binding pocket for the residues in the positions $C$-terminal to $\mathrm{pY}$ lies on the other side. The two R residues in $\alpha \mathrm{I}(\mathrm{R} \alpha \mid 2)$ and in $\beta \mathrm{B}(\mathrm{R} \beta \mathrm{B} 5)$ make key contact with $\mathrm{pY}$. This ribbon representation is redrawn based on the folded structure of the C-terminal SH2 domain in P85 $\alpha$ (PDB accession number: 1QAD). N, N-terminal end. (b) The positional distribution of $\mathrm{SH} 2$ mutations in pan-cancers. Analysis was made based on cancer genome data downloaded from the COSMIC database ([33], accessed 20 August 2017) and sequence alignment of the 23 SH2 domains having mutations in the 468 patients (Supplementary Document, page 15). The horizontal axis represents the peptide sequence containing the first 80 amino acids of the C-terminal instance of the SH2 domain in P85 $\alpha$. Structural elements are colored in the same way as in Panel a. (c) The SH2 mutations occurring in the 468 patients are from 23 proteins (Table S5). (d) The Kaplan-Meier survival analysis for the $\mathrm{SH} 2$ domain. The log rank $p$-value for 0.173

new biomarkers and new drug targets among 96 SH2containing proteins.

\section{Conclusions}

Our integrative analyses provide biological insights into the progression from in situ ductal carcinoma to metastatic cancer. It may assist determining biomarkers for early detection of IDBC, which can be a deciding factor between successful treatment and death. It can also be valuable in designing combinatorial therapeutic treatments defined at the network level for IDBC [37].

\section{Methods}

\section{Data collection}

The datasets for 468 patients with IDBC and 112 patients with ILBC were used in this study, which were deposited 
in the Phsae II of the TCGA project (http://tcga-data.nci. nih.gov/tcga/dataAccessMatrix.htm, download 1 March 2016). These datasets contain information on mutation (single nucleotide variants and copy number variants) and survival status of tumors (Table S1). The 468 patients with IDBC were originally staged using different versions of the American Joint Committee on Cancer TNM system in the past decade [10]. To keep consistency in stage information, we re-staged the cases using the seventh edition of the manual if they were staged using the sixth or earlier edition of it. Additionally, there were only 10 patients in Stage IV and thus we merged Stage IV into Stage III, obtaining 89, 287, and 92 cases in Stages I, II and III, respectively (Table $\mathrm{S} 1$ ).

\section{Survival analysis}

The SRUVIVAL R-program was used for the KaplanMeier and COX-PH analyses. The two survival analyses are fully documented in the Supplementary Document. Of note, $P$-value was computed by using the log-rank test in the Kaplan-Meier analysis and the Wald test in the Cox- $\mathrm{PH}$ analysis.

\section{Prioritization of mutated genes}

MutSigCV2 (Lawrence et al., 2014) was used to identify a list of 12 recurrently mutated genes discussed in the "Results" section. MutSigCV2 uses genomic covariates to account for the specific background mutation rate for each gene and uses both $P$-values and $q$-values for measuring the mutation significance. Like any bioinformatic tool for mutational analysis in cancer biology, the output from a MutSigCV2 analysis is sensitive to the input dataset [38]. We conducted a kind of saturated analysis [18], like bootstrap analysis, for further reducing false positive predictions. We created six extra datasets by randomly selecting 410, 420, 430, 440, 450 and 460 patients from the total of 468 patients. We then ran the program on the original dataset as well as the six derived ones, resulting in the final list of 12 significantly mutated genes via consensus (Table S2; Section 3.1 of the Supplementary Document).

\section{Mutational analyses of mutated protein domains}

Protein domain analysis was conducted using a method similar to the one in [27]. Mutations in the protein domain-coding regions in all genes containing a given protein domain are tallied to identify mutations that are rare at the gene level, but occur recurrently within the protein domain. Missense mutations were mapped onto the Pfam human protein domains (Pfam-A database, version 29; https://doi.org/ftp://ftp.ebi.ac.uk/, accessed 1 March 2017) (Fig. S2). The Human Pfam-A database contained 49,636 protein domains, 33,044 (sequence) families, 739 motifs, 9,238 repeats, 240 coiled-coils and 192 disorders.
Only protein domains with E-values $<10$ e-5 were used in our analysis. In total, 1,217 Pfam-A protein domains had mutations across 468 patients (Table S3).

We made two assumptions: (i) a mutation is uniformly distributed in each protein gene (i.e., a mutation hits the encoding region of a protein domain with a probability equal to the ratio of the domain length to the protein length and (ii) a mutation occurs independently in each position of a gene sequence. Under the two assumptions, the number $N$ of mutations occurring in the domainencoding regions of the respective gene members has a Poisson-binomial distribution, for which the exact $P$ value of the event that $N>$ \#(observed mutations) can be computed with a dynamic programming algorithm. Our computer program developed from this method is available upon request. It gave $P$-values that were more accurate than the permutation test [27], particularly when its true value was less than $10^{-7}$.

\section{Analysis of network and pathway alterations}

We used the HotNet2 program [19] to identify significant alterations in signaling pathways and protein complexes. HotNet2 was designed using an insulated heat diffusion process, which is actually a random walk with a restart, on a protein network to capture the mutation alterations within a local subnetwork around a protein.

\section{Supplementary information \\ Supplementary information accompanies this paper at https://doi.org/10.1186/s12920-020-00777-y.}

Additional file 1: Supplementary document for data analyses.

Additional file 2: Fig. S1-S6.

Additional file 3: Table S1: (Related to Fig. 1) The mutational and clinical information for 468 patients.

Additional file 4: Table S2: (Related to Table 1) The gene lists output from MutSigCV2.

Additional file 5: Table S3: (Related to Fig. 2) The 1,217 domains that mutated in IDBC.

Additional file 6: Table S4: (Related to Fig. 2) Functional annotation of the identified 22 protein domains.

Additional file 7: Table S5: (Related to Fig. 2) The identified 22 protein domains.

Additional file 8: Table S6: 11 protein complexes and signaling pathways identified by network enrichment analysis.

\section{Abbreviations}

COSMIC: ; ER+: estrogen receptor-positive; FDR: false discovery rate; HR: hazard ratio; HER2+: HER2 amplified; IDBC: invasive ductal breast cancer; ILBC: invasive lobular breast cancer; MC: mutation coverage; PR+: progesterone receptor-positive; PTK: protein tyrosine kinase; SH2: Src-homology 2; TCGA: The Cancer Genome Atlas

\section{Acknowledgments}

We thank Xuefeng Cui for assistance with protein structure, Weiwei Zai, M. Karthik and Q. Yu for critical comments on the first draft of this work. 


\section{About this supplement}

This article has been published as part of BMC Medical Genomics Volume 13 Supplement 10, 2020: Selected articles from the 18th Asia Pacific Bioinformatics Conference (APBC 2020): medical genomics. The full contents of the supplement are available online at https://bmcmedgenomics.biomedcentral.com/articles/ supplements/volume-13-supplement-10.

\section{Authors' contributions}

LXZ conceived the project. TY performed the computational and statistical analysis; KPC ESC, TY and LXZ studied the functions of the identified domains and signaling pathways. TY and LXZ wrote the article with help from ESC and KPC. All author(s) read and approved the final manuscript.

\section{Funding}

Publication costs of this work are funded by the National Research Fund [grant NRF2016NRF-NSFC001-026]. This work was also supported by Singapore MOE ARC grant MOE2014-T2-1-155. The funding body did not plat any role in the design of the study and collection, analysis, and interpretation of data and in writing the manuscript.

\section{Availability of data and materials}

Data sharing is not applicable to this article as no datasets were generated or analysed during the current study.

\section{Ethics approval and consent to participate}

This research did not involve any human subjects, human material, or human data. The ethics approval is not applicable.

\section{Consent for publication}

Not applicable.

\section{Competing interests}

The authors declare that they have no competing interests.

\section{Author details}

${ }^{1}$ Department of Mathematics, National University of Singapore, 10 Lower Kent Ridge Road, 119076 Singapore, Singapore. ${ }^{2}$ Department of Statistics and Applied Probability, National University of Singapore, 6 Science Drive 2 117546 Singapore, Singapore. ${ }^{3}$ Department of Biochemistry, National University of Singapore, 8 Medical Drive, 117596 Singapore, Singapore. ${ }^{4}$ Computational Biology Programme, National University of Singapore, 8 Medical Drive, 117596 Singapore, Singapore.

\section{Published: 22 October 2020}

\section{References}

1. American Cancer Society. Breast Cancer Facts and Figures 2015-2016. Atlanta: American Cancer Society; 2015.

2. van Zee KJ, Barrio AV, Tchou J. Treatment and long-term risks for patients with a diagnosis of ductal carcinoma in situ. JAMA Oncol. 2016;2:397-8.

3. Kaur H, Mao S, Shah S, Gorski DH, Krawetz SA, Sloane BF, et al. Next-generation sequencing: a powerful tool for the discovery of molecular markers in breast ductal carcinoma in situ. Expert Rev Mol Diagn. 2013;13:151-65.

4. Lesurf R, Aure MR, Mork HH, Vitelli V, Lundgren S, Borresen-Dale AL, Engebraten $\mathrm{O}$, et al. Molecular features of subtype-specific progression from ductal carcinoma in situ to invasive breast cancer. Cell Rep. 2016;16: 1166-79.

5. Miron A, Varadi M, Carrasco D, Li H, Luongo L, Kim HJ, Park SY, Cho EY, Lewis G, Kehoe S, Iglehart JD, Dillon D, Allred DC, Macconaill L, Gelman $\mathrm{R}$, Polyak K. PIK3CA mutations in in situ and invasive breast carcinomas. Cancer Res. 2010;70:5674-8.

6. Banerji S, Cibulskis K, Rangel-Escareno C, Brown KK, Carter SL, Frederick AM, Lawrence MS, Sivachenko AY, Sougnez C, Zou L, et al. Sequence analysis of mutations and translocations across breast cancer subtypes. Nature. 2012:486:405-9.

7. Pape-Zambito D, Jiang Z, Wu H, Devarajan K, Slater CM, Cai KQ, Patchefsky A, Daly MB, Chen X. Identifying a highly-aggressive DCIS subgroup by studying intra-individual DCIS heterogeneity among invasive breast cancer patients. PloS ONE. 2014;9:e100488.
8. Raphael BJ, Hruban RH, Aguirre AJ, Moffitt RA, Yeh JJ, Stewart C, Robertson AG, Cherniack AD, Gupta M, Getz G, et al. Integrated genomic characterization of pancreatic ductal adenocarcinoma. Cancer Cell. 2017;32:185-203.

9. Yates LR, Gerstung M, Knappskog S, Desmedt C, Gundem G, Van Loo P, Aas $\mathrm{T}$, Alexandrov LB, Larsimont D, Davies $\mathrm{H}$, et al. Subclonal diversification of primary breast cancer revealed by multiregion sequencing. Nat Med. 2015;21:751-9.

10. Edge SB, Compton CC. The American Joint Committee on Cancer: the 7th edition of the AJCC cancer staging manual and the future of TNM. Ann Surg Oncol. 2010;17:1471-4.

11. Andersen J, Poulsen HS. Immunohistochemical estrogen receptor determination in paraffin-embedded tissue. Prediction of response to hormonal treatment in advanced breast cancer. Cancer. 1989;64:1901-8.

12. Perou CM, Sorlie T, Eisen MB, van de Rijn M, Jeffrey SS, Rees CA, Pollack $J R$, Ross DT, Johnsen $H$, Akslen LA, et al. Molecular portraits of human breast tumors. Nature. 2000;406:747-52.

13. The Cancer Genome Atlas Network. Comprehensive molecular portraits of human breast tumors. Nature. 2012;490:61-70.

14. Stephens PJ, Tarpey PS, Davies H, Van Loo P, Greenman C, Wedge DC, Nik-Zainal S, Martin S, Varela I, Bignell GR, et al. The landscape of cancer genes and mutational processes in breast cancer. Nature. 2012;486:400-4.

15. Nik-Zainal S, Davies H, Staaf J, Ramakrishna M, Glodzik D, Zou X, Martincorena I, Alexandrov LB, Martin S, Wedge DC, et al. Landscape of somatic mutations in 560 breast cancer whole-genome sequences. Nature. 2016;534:47-54

16. Creixell P, Reimand J, Haider $S, W u G$, Shibata T, Vazquez M, Mustonen V, Gonzalez-Perez A, Pearson J, Sander C, et al. Pathway and network analysis of cancer genomes. Nat Methods. 2015;12:615-21.

17. Mertins P, Mani DR, Ruggles KV, Gillette MA, Clauser KR, Wang P, Wang X, Qiao JW, Cao S, Petralia F, et al. Proteogenomics connects somatic mutations to signalling in breast cancer. Nature. 2016;534:55-62.

18. Lawrence MS, Stojanov P, Mermel CH, Robinson JT, Garraway LA, Golub TR, Meyerson M, Gabriel SB, Lander ES, Getz G. Discovery and saturation analysis of cancer genes across 21 tumour types. Nature. 2014;505: 495-501.

19. Leiserson MDM, Vandin F, Wu H-T, Dobson JR, Eldridge JV, Thomas JL, Papoutsaki A, Kim Y, Niu B, McLellan M, et al. Pan-cancer network analysis identifies combinations of rare somatic mutations across pathways and protein complexes. Nat Genet. 2015;47:106-14.

20. Rudolph JD, de Graauw M, van de Water B, Geiger T, Sharan R. Elucidation of signaling pathways from large-scale phosphoproteomic data using protein interaction networks. Cell Syst. 2016;3:585-93.

21. Cowen L, Ideker T, Raphael BJ, Sharan R. Network propagation: a universal amplifier of genetic associations. Nat Rev Genet. 2017;18:551-62.

22. Muggerud AA, Hallett M, Johnsen H, Kleivi K, Zhou W, Tahmasebpoor S, Amini R-M, Botling J, Børresen-Dale A-L, Sørlie T, et al. Molecular diversity in ductal carcinoma in situ (DCIS) and early invasive breast cancer. Mol Oncol. 2010;4:357-68.

23. Futreal PA, Coin $L$, Marshall $M$, Down $T$, Hubbard $T$, Wooster R, Rahman N, Stratton MR. A census of human cancer genes. Nat Rev Cancer. 2004;4: $177-83$.

24. Hurtado A, Holmes KA, Geistlinger TR, Hutcheson IR, Nicholson RI, Brown M, Jiang J, Howat W, Ali S, Carroll J. Regulation of ERBB2 by oestrogen receptor-PAX2 determines response to tamoxifen. Nature. 2008;456:663-6.

25. Otto T, Sicinski P. Cell cycle proteins as promising targets in cancer therapy. Nat Rev Cancer. 2017;17:93-115.

26. Nehrt NL, Peterson TA, Park D, Kann MG. Domain landscapes of somatic mutations in cancer. BMC Genomics. 2012;13:S9.

27. Miller ML, ReznikE, Gauthier NP, Aksoy BA, Korkut A, Gao J, Ciriello G, Schultz N, Sander C. Pan-cancer analysis of mutation hotspots in protein domains. Cell Syst. 2015;1:197-209.

28. Yang F, Petsalaki E, Rolland T, Hill DE, Vidal M, Roth FP. Protein domain-level landscape of cancer-type-specific somatic mutations. PLoS Comput Biol. 2015;11:e1004147.

29. Kamal M, Shaaban AM, Zhang L, Walker C, Gray S, Thakker N, Toomes C, Speirs V, Bell S, et al. Loss of CSMD1 expression is associated with high tumor grade and poor survival in invasive ductal breast carcinoma. Breast Cancer Res Treat. 2010;121:555-63. 
30. Nooter K, De La Riviere GB, Look MP, van Wingerden KE,

Henzen-Logmans SC, Scheper RJ, Flens MJ, Klijn JGM, Stoter G, Foekens

$J A$, et al. The prognostic significance of expression of the multidrug

resistance-associated protein (MRP) in primary breast cancer. British J Cancer. 1997;76:486-93.

31. Pawson T, Gish GD, Nash P. SH2 domains, interaction modules and cellular wiring. Trends Cell Biol. 2001;11:504-11.

32. Eck MJ, Shoelson SE, Harrison SC. Recognition of a high-affinity phosphotyrosyl peptide by the Src homology-2 domain of p56lck. Nature. 1993;362:87-91.

33. Forbes $\mathrm{SA}$, Beare D, Boutselakis $\mathrm{H}$, Bamford $\mathrm{S}$, Bindal N, Tate J, et al. COSMIC: somatic cancer genetics at high-resolution. Nucleic Acids Res. 2016:45:D777-83.

34. Saffran DC, Parolini O, Fitch-Hilgenberg ME, Rawlings DJ, Afar D, Witte $\mathrm{ON}$, Conley ME. A point mutation in the SH2 domain of Bruton's tyrosine kinase in atypical X-linked agammaglobulinemia. New England J Med. 1994;330:1488-91.

35. Tartaglia M, Mehler EL, Goldberg R, Zampino G, Brunner HG, Kremer H, van der Burgt I, Crosby A, Ion A, Jeffery S, Kalidas K, Patton M, Kucherlapati R, Gelb B. Mutations in PTPN11, encoding the protein tyrosine phosphatase SHP-2, cause Noonan syndrome. Nat Genet. 2001;29:465-8.

36. Ciriello G, Gatza ML, Beck AH, Wilkerson MD, Rhie SK, Pastore A, et al. Comprehensive molecular portraits of invasive lobular breast cancer. Cell. 2015;163:506-19.

37. Jackson RA, Chen ES. Synthetic lethal approaches for assessing combinatorial efficacy of chemotherapeutic drugs. Pharmacol Ther. 2016;162:69-85.

38. Tokheim CJ, Papadopoulos N, Kinzler KW, Vogelstein B, Karchin R. Evaluating the evaluation of cancer driver genes. Proc Natl Acad Sci U S A. 2016;113:14330-5.

\section{Publisher's Note}

Springer Nature remains neutral with regard to jurisdictional claims in published maps and institutional affiliations.

Ready to submit your research? Choose BMC and benefit from:

- fast, convenient online submission

- thorough peer review by experienced researchers in your field

- rapid publication on acceptance

- support for research data, including large and complex data types

- gold Open Access which fosters wider collaboration and increased citations

- maximum visibility for your research: over $100 \mathrm{M}$ website views per year

At BMC, research is always in progress.

Learn more biomedcentral.com/submissions 